\title{
Prüf-Falle Plausizeiten
}

\section{Vorsicht beim Quartalsprofil!}

\author{
Für fast alle Leistungen sind im Anhang des EBM Prüfzeiten angege- \\ ben. Mit ihnen lassen sich Tages- und Quartalsprofile der Arbeitszeit \\ von Vertragsärzten erstellen. Vor allem bei den Quartalsprofilen droht \\ großen Praxen mit vielen chronisch kranken Patienten Ärger. Doch der \\ lässt sich vermeiden.
}

$\mathrm{P}$ ost für die Praxis von der Kassenärztlichen Vereinigung (KV): Auf den Tisch flattert die Ankündigung einer Abrechnungsprüfung - wegen Überschreitung des „Zeitprofils“. Grund: Das Quartalszeitprofil von maximal 780 Stunden ist überschritten, die Tageszeitprofile jedoch liegen alle im Normbereich, das heißt unter zwölf Stunden.

\section{Prüf- und Kalkulationszeit sind zu unterscheiden}

Doch wie kann ein Arzt im Quartal zu viel arbeiten, obwohl er an keinem Tag länger als zwölf Stunden tätig war? Zur Erinnerung: Für (fast) jede Leistung ist im Einheitlichen Bewertungsmaßstab (EBM) eine Zeitdauer hinterlegt, die dafür in der Regel benötigt wird - und zwar differenziert nach Tages- und nach Quartalsprofil. Diese Zeiten sind im Anhang 3 des EBM nachzulesen. Dort finden sich die Angaben für den ,zur Leistungserbringung erforderlichen Zeitaufwand des Vertragsarztes (...)“. Dabei sind zwei Kategorien zu unterscheiden:

1. Zum einen ist das die obligatorisch gemäß Leistungslegende abzuleistende Zeit, also die Mindestzeit, in der Tabelle in Anhang 3 als „Prüfzeit“ bezeichnet. Das sind Zeitansätze, die in den EBM-Legenden als Mindestdauer für die Leistungserbringung angegeben sind. Bei der Gesprächsleistung für Hausärzte nach der Gebührenordnungsposition (GOP) 03230 sind zum Beispiel die obligaten zehn Minuten als Prüfzeit angesetzt. Sie gehen sowohl ins Tages- als auch ins Quar- talsprofil ein. Es gibt aber auch Leistungspauschalen, die ausschließlich ins Quartalsprofil eingehen, für die es also Prüfzeiten nur im Anhang 3 gibt, nicht jedoch unmittelbar in den Leistungslegenden, beispielsweise die Versichertenpauschale oder die Chronikerzuschläge für Hausärzte.

2.Zum zweiten gibt es die Kalkulationszeit: Sie ist derjenige Zeitaufwand, der zur ordnungsgemäßen Erfüllung des Leistungsinhalts im Durchschnitt angesetzt (einkalkuliert) wird. Sie bildet damit letztlich auch die Basis für die Bewertung der Leistung. Die Kalkulationszeit spielt für die Plausibilitätsprüfung aber keine Rolle.

\section{Ursachen für die Überschreitung von Zeitprofilen}

Für die Plausiprüfung entscheidend ist die Prüfzeit einer Leistung. Jede erbrachte und in der Abrechnung notierte Leistung geht mit der vorgegebenen Prüfzeit in das Zeitprofil ein. Damit sind die Zeitprofile letztlich Resultat einer simplen Addition: Die Praxis-EDV addiert bei jeder notierten Leistung die jeweils vorgegebenen Minuten hinzu. Wer diese Funktion seiner EDV nutzt, behält also stets in etwa den Überblick - jedenfalls, wenn die Leistungspositionen direkt nach Erbringung notiert werden.

Nützlich ist es dennoch, zu verstehen, wie die Überschreitung von Zeitprofilen zustande kommen kann: Für das Tageszeitprofil sind zwölf Stunden vorgegeben, für das Quartalszeitprofil 780 Stunden. Dabei wird von einer 5-Tagewoche ausgegangen: Das Quartal hat im
Schnitt 65 Arbeitstage verteilt auf 13 Wochen. Der Verordnungsgeber geht davon aus, dass ein Vertragsarzt maximal fünf Tage pro Woche arbeitet. Wie kann es sein, dass ein Arzt bei Einhaltung der Zwölf-Stunden-Grenze für die Tagesarbeitszeit, im Quartalsprofil die 780 Stunden überschreitet? Eine Möglichkeit ist die Samstag-Sprechstunde: Wer bei sonst „normalem“ Tagesablauf mit zwölf Stunden Arbeitszeit diese zusätzlich anbietet, der hat mit dem Tageszeitprofil von zwölf Stunden kein Problem. Er überschreitet jedoch das Quartalsprofil, da er bei etwa drei Sprechstunden am Samstag circa 40 Stunden mehr im Quartal hat und somit auf rund 820 Stunden kommt.

Wichtiger noch: Wie oben beschrieben, gibt es manche Leistungen, die nur ins Quartalsprofil eingehen, weil sie pauschal für viele Tätigkeiten berechnet werden, die im Laufe eines Quartals erbracht werden, zum Beispiel die Versichertenpauschale und die Grundpauschale. Eine Praxis mit einer sehr hohen Fallzahl, verbunden mit sehr vielen chronisch kranken Patienten bekommt daher leicht ein Problem. Denn allein mit der Berechnung der Versicherten-/ Grundpauschale plus der Chronikerpauschale im hausärztlichen Bereich oder der Zusatzpauschale für die Betreuung eines Patienten mit einer malignen Erkrankung, liegt eine Praxis mit 2.000 Patienten schon fast über der 780 -Stunden-Grenze. Dabei sind noch keine anderen Leistungen mit berücksichtigt.

$\mathrm{Zu}$ bedenken ist: Die Plausizeiten sind letztlich nur ein Aufgreifkriterium für die Wirtschaftlichkeitsprüfung. Wer mittels guter Dokumentation nachweisen kann, dass alle abgerechneten Leistungen auch tatsächlich erbracht wurden, der muss nichts befürchten. Wer Ärger vermeiden will, sollte rechtzeitig die Addition der Zeiten im Blick behalten.

Dr. Dr. Peter Schlüter 